hep-ph/0004068

DESY-00-057

Freiburg-THEP 00/6

April 2000

\title{
Heavy Quark Pair Production in Polarized Photon-Photon Collisions
}

\author{
G. Jikiaウ" \\ Fakultät für Physik, \\ Albert-Ludwigs-Universität Freiburg, \\ Hermann-Herder-Str. 3, D-79104 Freiburg, Germany
}

A. Tkabladze

DESY Zeuthen, D-15738, Zeuthen, Germany

\begin{abstract}
We present the next-to-leading-order cross sections of the heavy quark-antiquark pair production in polarized photon-photon collision for the general case of photon polarizations. The numerical results for top-antitop production cross sections together with production asymmetries are obtained for linearly polarized photon-photon collisions, including one-loop QCD radiative corrections.
\end{abstract}

PACS 12.38.Bx, 13.88.+e, 14.65.Ha

\footnotetext{
${ }^{\dagger}$ On leave of absence from IHEP, 142284 Protvino (Moscow Region), Russian Federation
} 


\section{Introduction}

The experimental discovery of the Higgs boson is crucial for the understanding of the mechanism of electroweak symmetry breaking. The search for Higgs particles is one of the main goals for the LEP2 and Tevatron experiments and is one of the major motivations for the future Large Hadron Collider (LHC) and Linear $e^{+} e^{-}$Collider (LC). Once the Higgs boson is discovered, it will be of primary importance to determine in a model independent way its tree-level and one-loop induced couplings, spin, parity, $C P$-nature, and its total width. In this respect the $\gamma \gamma$ Compton Collider [1] option of the LC offers a unique opportunity to produce both Standard Model (SM) Higgs boson and neutral Higgs states $h, H, A$ of the Minimal Supersymmetric Standard Model (MSSM) or general two Higgs Doublet Model (2HDM) [2] as $s$-channel resonance decaying into $b \bar{b}, W W^{*}, Z Z$ or $t \bar{t}$ :

$$
\gamma \gamma \rightarrow h^{0}, H^{0}, A^{0} \rightarrow b \bar{b}, W W^{*}, Z Z, t \bar{t} .
$$

The ability to control the polarizations of back-scattered photons [1] provides a powerful means for exploring the $C P$ properties of any single neutral Higgs boson that can be produced with reasonable rate at the Photon Linear Collider [3]. $C P$-even Higgs $0^{++}$bosons $h^{0}, H^{0}$ couple to the combination

$$
\overrightarrow{\varepsilon_{1}} \cdot \overrightarrow{\varepsilon_{2}}=-\frac{1}{2}\left(1+\lambda_{1} \lambda_{2}\right)
$$

while a $C P$-odd $0^{-+}$Higgs boson $A^{0}$ couples to

$$
\left[\overrightarrow{\varepsilon_{1}} \times \overrightarrow{\varepsilon_{2}}\right] \cdot \overrightarrow{k_{\gamma}}=\frac{\omega_{\gamma}}{2} i \lambda_{1}\left(1+\lambda_{1} \lambda_{2}\right)
$$

where $\overrightarrow{\varepsilon_{i}}$ and $\lambda_{i}$ are photon polarization vectors and helicities. The first of these structures couples to linearly polarized photons with the maximal strength if the polarizations are parallel, the letter if the polarizations are perpendicular. Moreover, if the Higgs boson is a mixture of $C P$-even and $C P$-odd states, as can occur e.g. in a general $2 \mathrm{HDM}$ with $C P$-violating neutral sector, the interference of these two terms gives rise to $C P$-violating asymmetries [3]. Since MSSM Higgs particles $h^{0}, H^{0}, A^{0}$ decay predominantly into $b \bar{b}$ or $t \bar{t}$ quark pairs depending on the mass of the Higgs boson, the heavy quark pair background in $\gamma \gamma$ collisions has been studied in great detail. One-loop QCD corrections were calculated for the photon helicity states corresponding to projection of total angular momentum on beam axes $J_{z}=0$ and $J_{z}= \pm 2$ [5, 6]. Virtual one-loop QCD corrections for $J_{z}=0$ were found to be especially large due to the double-logarithmic enhancement factor, so that the corrections are comparable or even larger than the Born contribution for the two-jet final topologies [5]. In order to solve this theoretical problem leading QCD corrections for $J_{z}=0$ have been calculated at the two-loop level [7] and recently these leading double-logarithmic QCD corrections were resummed to all orders 8]. The account of non-Sudakov form factor to higher orders makes the $J_{z}=0$ cross section well defined and positive definite in all regions of the phase space [8]. All these studies of the influence of QCD corrections on heavy quark production in $\gamma \gamma$ collisions were concentrated on circularly polarized initial photons. However, for the direct measurements of the parity of states of Higgs bosons, (112), linear polarization of photon beams is needed [3].

In the present paper we consider the QCD corrections to heavy quark-antiquark pair production in photon-photon collision for the general case of initial photon polarizations. We mainly concentrate on QCD corrections for linearly polarized photon-photon collisions. The production cross sections and spin asymmetries for $t \bar{t}$-pair production are calculated for linearly polarized

photon collisions. The measurement of spin asymmetries is necessary to determine the $C P$ parity 
of the Higgs boson. In the scattering of linearly polarized photon off circularly polarized ones at one-loop level azimuthal asymmetries arise in the production of heavy fermion pairs. This is a pure quantum effect which does not exist at the Born level. These type of asymmetries are suppressed by factor $m_{Q}^{2} / s, s$ is the c.m.s energy of colliding photons, and are sizeable only for $t \bar{t}$-pair production.

The paper is organized as follows. In the next Section we recall the basic definitions and consider the Born cross section of heavy quark-antiquark pair production in polarized photon collisions. Calculations of virtual corrections are presented in Section 3. The real gluon emission part is discussed in Section 4. The numerical results for top-antitop pair production cross sections and expected asymmetries for linearly polarized photon beams are discussed in Section 5 .

\section{Born Cross Section}

The cross section of heavy quark-antiquark pair production in polarized photon-photon collision

$$
\gamma\left(p_{1}\right)+\gamma\left(p_{2}\right) \rightarrow Q\left(p_{3}\right)+\bar{Q}\left(p_{4}\right)
$$

can be written in the most general form using the Stokes parameters which describe the polarizations of initial photons. The covariant density matrix of polarized photon with arbitrary polarization can be written in the following form

$$
\begin{aligned}
\rho_{\mu \nu}^{(1,2)}= & \frac{1}{2}\left(e_{\mu}^{x} e_{\nu}^{x}+e_{\mu}^{y} e_{\nu}^{y}\right) \pm \frac{\xi_{1}^{(1,2)}}{2}\left(e_{\mu}^{x} e_{\nu}^{y}+e_{\mu}^{y} e_{\nu}^{x}\right) \\
& \mp \frac{\mathrm{i} \xi_{2}^{(1,2)}}{2}\left(e_{\mu}^{x} e_{\nu}^{y}-e_{\mu}^{y} e_{\nu}^{x}\right)+\frac{\xi_{3}^{(1,2)}}{2}\left(e_{\mu}^{x} e_{\nu}^{x}-e_{\mu}^{y} e_{\nu}^{y}\right) .
\end{aligned}
$$

Here $\xi_{i}^{(1,2)}$ are three Stokes parameters describing polarization of the photon with momentum $p_{1,2}$ and $e^{x}$ and $e^{y}$ denote ort vectors in $x$ and $y$ directions.

The momenta of the particles involved in the reaction in the c.m.s. of the initial photons are given by

$$
\begin{gathered}
p_{1}=E(1 ; 0,0,1), \quad p_{2}=E(1 ; 0,0,-1), \\
p_{3}=E(1 ; \beta \sin \theta \cos \phi, \beta \sin \theta \sin \phi, \beta \cos \theta), \quad p_{4}=E(1 ;-\beta \sin \theta \cos \phi,-\beta \sin \theta \sin \phi,-\beta \cos \theta),
\end{gathered}
$$

where $E=\sqrt{s} / 2$ is the photon beam energy and $\beta=\sqrt{1-4 m_{Q}^{2} / s}$ is the quark (antiquark) velocity.

With this definitions, the Born cross section of heavy quark pair production in photon-photon collisions has the form

$$
\begin{aligned}
\frac{\mathrm{d} \sigma(\gamma \gamma \rightarrow b \bar{b})}{\mathrm{d} t}= & \frac{6 \pi \alpha^{2} e_{Q}^{4}}{s^{2}}\left\{\left(\frac{t_{1}}{u_{1}}+\frac{u_{1}}{t_{1}}\right)\left(1+\tilde{\xi}_{2}^{(1)} \tilde{\xi}_{2}^{(2)}\left(1-2 \frac{Y}{t_{1} u_{1}}\right)\right)+4\left(1+\xi_{3}^{(1)}+\tilde{\xi}_{3}^{(2)}\right) \frac{m_{Q}^{2} s Y}{t_{1}^{2} u_{1}^{2}}\right. \\
& \left.+2\left(\tilde{\xi}_{1}^{(1)} \tilde{\xi}_{1}^{(2)}-\tilde{\xi}_{3}^{(1)} \tilde{\xi}_{3}^{(2)}\right)\left(1-2 \frac{Y}{t_{1} u_{1}}\right)-4 \tilde{\xi}_{3}^{(1)} \tilde{\xi}_{3}^{(2)} \frac{Y^{2}}{t_{1}^{2} u_{1}^{2}}\right\}
\end{aligned}
$$

where

$$
Y=t u-m_{Q}^{4}, \quad t_{1}=t-m_{Q}^{2} \quad \text { and } \quad u_{1}=u-m_{Q}^{2}
$$

and $s, t$ and $u$ are Mandelstam variables for the process under consideration,

$$
s=\left(p_{1}+p_{2}\right)^{2}, \quad t=\left(p_{1}-p_{3}\right)^{2}, \quad u=\left(p_{1}-p_{4}\right)^{2} .
$$


In the above formulae instead of the original Stokes parameters their combinations are used. The expressions of the cross sections are shorter and more convenient for integration if one includes the dependence on the azimuthal angle in the Stokes parameters, $\tilde{\xi}_{i}^{(1,2)}$. They can be expressed by original Stokes parameters through the equations:

$$
\begin{aligned}
& \tilde{\xi}_{1}^{(1)}=\xi_{1}^{(1)} \cos (2 \phi)-\xi_{3}^{(1)} \sin (2 \phi), \\
& \tilde{\xi}_{1}^{(1)}=\xi_{2}^{(1)} \\
& \tilde{\xi}_{3}^{(1)}=\xi_{1}^{(1)} \sin (2 \phi)+\xi_{3}^{(1)} \cos (2 \phi), \\
& \tilde{\xi}_{1}^{(2)}=\xi_{1}^{(2)} \cos (2 \phi)+\xi_{3}^{(2)} \sin (2 \phi), \\
& \tilde{\xi}_{1}^{(2)}=\xi_{2}^{(2)} \\
& \tilde{\xi}_{3}^{(2)}=-\xi_{1}^{(2)} \sin (2 \phi)+\xi_{3}^{(2)} \cos (2 \phi),
\end{aligned}
$$

here $\phi$ is the azimuthal angle. Parameters $\tilde{\xi}_{i}^{(1,2)}$ describe photon polarization with respect to unit vectors $\tilde{e^{x}}, \tilde{e^{y}}$, where $\tilde{e^{x}}$ lies in the reaction plane and $\tilde{e^{y}}$ is orthogonal to the reaction plane.

\section{Virtual Corrections}

The first order QCD corrections to the cross section are determined by the interference between the tree level and one-loop diagrams. At the one-loop level no three-gluon vertex enters and the calculation is analogous to calculations of QED corrections to Compton scattering for finite electron mass (see e.g. [9]). The calculations of virtual corrections were done by using the symbolic manipulation program FORM [10]. To regularise the infrared singularities we introduced an infinitesimal mass of the gluon $\lambda$. In the basis of the Stokes parameters the one-loop corrections have the form

$$
\begin{aligned}
\frac{\mathrm{d} \sigma(\gamma \gamma \rightarrow Q \bar{Q})}{\mathrm{d} t}= & \mathcal{M}_{0}+\tilde{\xi}_{1}^{(1)} \tilde{\xi}_{1}^{(2)} \mathcal{M}_{11}+\mathrm{i}\left(\tilde{\xi}_{1}^{(1)} \tilde{\xi}_{2}^{(2)}+\tilde{\xi}_{2}^{(1)} \tilde{\xi}_{1}^{(2)}\right) \mathcal{M}_{12}+\tilde{\xi}_{2}^{(1)} \tilde{\xi}_{2}^{(2)} \mathcal{M}_{22}+\tilde{\xi}_{3}^{(1)} \tilde{\xi}_{3}^{(2)} \mathcal{M}_{33} \\
& +\left(\tilde{\xi}_{3}^{(1)}+\tilde{\xi}_{3}^{(2)}\right) \mathcal{M}_{03}
\end{aligned}
$$

In addition to the Born level expression, Eq. 2, there is the new term proportional to the nondiagonal product $\left(\xi_{1}^{(1)} \xi_{2}^{(2)}+\xi_{2}^{(1)} \xi_{1}^{(2)}\right)$, which corresponds to the scattering of linearly polarized photon on the circularly polarized one.

The functions $\mathcal{M}_{i}$ can be expressed through scalar one-loop integrals $B, C$ and $D$.

$$
\begin{aligned}
\mathcal{M}_{0}= & \frac{\alpha_{S} \alpha^{2} e_{Q}^{4}}{2 \pi s} \beta\left\{2 D(s, t)\left(-2 s \frac{s^{2} t_{1}+4 m_{Q}^{4}(s-2 t)}{t_{1} u_{1}}+s t-s s_{1}+8 m_{Q}^{4}\right)\right. \\
& -4 C_{1}(t)\left(2 \frac{3 m_{Q}^{4}-s^{2}-m_{Q}^{2} s}{u_{1}}+2 m_{Q}^{4} \frac{s-t_{1}}{t_{1}^{2}}-5 m_{Q}^{2}-s+t\right) \\
& +2 B(t)\left(4 m_{Q}^{4}\left(\frac{s+4 s_{1}}{s t_{1} u_{1}}-\frac{s_{0}}{t_{1}^{3}}\right)+4 m_{Q}^{2} \frac{3 s t-2 m_{Q}^{2} s_{1}}{s t_{1}^{2}}\right. \\
& \left.-\left(\frac{m_{Q}^{2}}{t}-\frac{s}{t_{1}}\right)\left(1-2 \frac{t_{2}}{u_{1}}\right)-2 \frac{s s_{1}}{u_{1} t_{1}}+1\right) \\
& +C_{1}(s) s_{0} \frac{s^{2}+2 m_{Q}^{2} s+2 t_{1} u_{1}}{t_{1} u_{1}}-C(s) s \frac{3 s^{2}-2 t_{1} u_{1}-8 m_{Q}^{4}}{t_{1} u_{1}}
\end{aligned}
$$




$$
\begin{aligned}
& +4 B(s) \frac{s Y}{s_{0} t_{1} u_{1}}+2 \ln \left(\frac{\lambda^{2}}{m_{Q}^{2}}\right)\left(4 \frac{m_{Q}^{4} s^{2}}{t_{1}^{2} u_{1}^{2}}-s \frac{s+4 m_{Q}^{2}}{t_{1} u_{1}}+2\right)+16 \frac{s^{2} m_{Q}^{4}}{t_{1}^{2} u_{1}^{2}} \\
& +4 \frac{m_{Q}^{4}}{t_{1} u_{1}}-m_{Q}^{2}(t+u) \frac{2 m_{Q}^{4}+t_{1} u_{1}}{t t_{1} u u_{1}}-4 m_{Q}^{2} s \frac{t_{1}^{2}+u_{1}^{2}}{t_{1}^{2} u_{1}^{2}}-3 s \frac{6 m_{Q}^{2}+s}{t_{1} u_{1}} \\
& +6+(t \leftrightarrow u)\} \\
& \mathcal{M}_{11}=\frac{\alpha_{S} \alpha^{2} e_{Q}^{4}}{2 \pi s} \beta\left\{2 D ( s , t ) \left(2 s \frac{8 m_{Q}^{4}+3 s^{2}-7 m_{Q}^{2} s}{u_{1}}+\frac{s^{2}}{Y}\left(s\left(s+m_{Q}^{2}-7 t\right)\right.\right.\right. \\
& \left.\left.+2 m_{Q}^{2}\left(3 t-m_{Q}^{2}\right)+\frac{s^{3}}{u_{1}}\right)+s t-3 m_{Q}^{2} s+8 m_{Q}^{4}\right) \\
& -4 C_{1}(t)\left(s \frac{s\left(s_{0}-5 t_{1}\right) u_{1}+4 m_{Q}^{2} t_{1} u_{1}+s^{3}}{u_{1} Y}+4 \frac{s^{2}-2 m_{Q}^{2} s+2 m_{Q}^{4}}{u_{1}}-5 m_{Q}^{2}+t\right) \\
& +2 B(t)\left(8 \frac{m_{Q}^{2} t}{t_{1}^{2}}-5 \frac{m_{Q}^{2} s}{t_{1} u_{1}}+\frac{m_{Q}^{2} Y}{t t_{1} u_{1}}-2 \frac{s}{u_{1}}+1\right) \\
& +C_{1}(s)\left(8 s^{2} \frac{s-2 m_{Q}^{2}}{Y}-\frac{s^{5}}{t_{1} u_{1} Y}-2 s \frac{3 s^{2}-5 m_{Q}^{2} s+4 m_{Q}^{4}}{t_{1} u_{1}}+2 s_{0}\right) \\
& -C(s) s\left(4 \frac{s^{2}+6 m_{Q}^{4}-3 m_{Q}^{2} s}{t_{1} u_{1}}-2 s \frac{3 s-4 m_{Q}^{2}}{Y}+\frac{s^{4}}{t_{1} u_{1} Y}+2\right) \\
& +2 B(s) s \frac{2 t_{1} u_{1}-s^{2}+2 m_{Q}^{2} s}{t_{1} u_{1} s_{0}}+4 \ln \left(\frac{\lambda^{2}}{m_{Q}^{2}}\right)\left(1-2 \frac{m_{Q}^{2} s}{t_{1} u_{1}}\right) \\
& \left.+\frac{m_{Q}^{4}}{t_{1} u}+\frac{m_{Q}^{4}}{t u_{1}}-19 \frac{m_{Q}^{2} s}{t_{1} u_{1}}+6+(t \leftrightarrow u)\right\} \\
& \mathcal{M}_{12}=\frac{\alpha_{S} \alpha^{2} e_{Q}^{4}}{2 \pi s} \beta\left\{2 D(s, t) m_{Q}^{2} s_{0}\left(2-\frac{s s_{0} t_{1}}{u_{1} Y}\right)\right. \\
& -4 C_{1}(t)\left(s_{0} \frac{t s-2 m_{Q}^{2} t_{1}}{Y}-\frac{\left(s-3 m_{Q}^{2}\right)^{2}}{u_{1}}-m_{Q}^{2} \frac{m_{Q}^{2} t_{1}-t s}{t_{1}^{2}}\right) \\
& +2 B(t) m_{Q}^{2}\left(\frac{\left(t_{1}+2 m_{Q}^{2}\right)\left(5 t_{1}-s\right)}{t_{1}^{3}}+\frac{\left((u-2 t) t_{1}+2 m_{Q}^{4}\right.}{t t_{1} u_{1}}\right) \\
& +C_{1}(s) s s_{0}\left(\frac{s-2 m_{Q}^{2}}{t_{1} u_{1}}-\frac{s_{0}}{Y}\right)+C(s)\left(s_{0}^{2} s\left(\frac{1}{t_{1} u_{1}}-\frac{1}{Y}\right)-8 \frac{m_{Q}^{2} Y}{t_{1} u_{1}}\right) \\
& \left.-Y \frac{2(t+u) m_{Q}^{2}-(t-u)^{2}}{t t_{1} u u_{1}}+(t \leftrightarrow u)\right\} \\
& \mathcal{M}_{22}=\frac{\alpha_{S} \alpha^{2} e_{Q}^{4}}{2 \pi s} \beta\left\{2 D(s, t)\left(2 s \frac{s^{2} t+m_{Q}^{2} s^{2}+4 m_{Q}^{4}(u-t)}{t_{1} u_{1}}+s\left(s-t-m_{Q}^{2}\right)-8 m_{Q}^{4}\right)\right. \\
& -4 C_{1}(t)\left(2 \frac{s^{2}+m_{Q}^{2} s-5 m_{Q}^{4}}{u_{1}}-2 \frac{m_{Q}^{4}\left(t_{1}-s\right)}{t_{1}^{2}}-t+s+5 m_{Q}^{2}\right)
\end{aligned}
$$




$$
\begin{aligned}
& +2 B(t)\left(2 m_{Q}^{2} \frac{4 t-u+3 m_{Q}^{2}}{t_{1} u_{1}}-4 m_{Q}^{2} \frac{3 s t-m_{Q}^{2} t_{1}}{t_{1}^{3}}+3 \frac{m_{Q}^{2} t_{1}-s t}{t t_{1}}-2 \frac{m_{Q}^{2} u}{t u_{1}}-1\right) \\
& -C_{1}(s)\left(\frac{s s_{0}\left(s+2 m_{Q}^{2}\right)}{t_{1} u_{1}}+2 s_{0}\right)-C(s)\left(3 s \frac{s^{2}-8 m_{Q}^{4}}{t_{1} u_{1}}-2 s\right) \\
& -4 B(s) \frac{s Y}{s_{0} t_{1} u_{1}}+2 \ln \left(\frac{\lambda^{2}}{m_{Q}^{2}}\right)\left(s \frac{s+4 m_{Q}^{2}}{t_{1} u_{1}}-2 \frac{m_{Q}^{2} s^{3}}{t_{1}^{2} u_{1}^{2}}-2\right) \\
& -s \frac{8 m_{Q}^{2} s^{2}-18 m_{Q}^{2} t_{1} u_{1}-3 t_{1} s u_{1}}{t_{1}^{2} u_{1}^{2}}+m_{Q}^{2}(u+t) \frac{2 m_{Q}^{4}+t_{1} u_{1}}{t t_{1} u u_{1}}-4 m_{Q}^{4} t_{1} u_{1} \\
& -6+(t \leftrightarrow u)\},
\end{aligned}
$$

$$
\begin{aligned}
\mathcal{M}_{33}= & \frac{\alpha_{S} \alpha^{2} e_{Q}^{4}}{2 \pi s} \beta\left\{-2 D(s, t)\left(\frac{s^{4} t_{1}}{u_{1} Y}+s^{2} \frac{7 t s_{1}-4 m_{Q}^{2} t_{1}-m_{Q}^{2}(s+t)}{Y}\right.\right. \\
& \left.-2 s \frac{t_{1}\left(3 s^{2}+m_{Q}^{4}\right)-7 m_{Q}^{2} t_{1} s_{1}-4 m_{Q}^{4} s}{t_{1} u_{1}}-16 \frac{s m_{Q}^{6}}{t_{1} u_{1}}+3 s m_{Q}^{2}-s t-8 m_{Q}^{4}\right) \\
& +4 C_{1}(t)\left(\frac{s^{3} t_{1}}{u_{1} Y}-4 \frac{\left(s-m_{Q}^{2}\right)^{2}+m_{Q}^{4}}{u_{1}}+\frac{5 s^{2} t_{1}+4 m_{Q}^{2} s\left(u_{1}+2 s\right)}{Y}+5 m_{Q}^{2}-t\right) \\
& +2 B(t)\left(\frac{\left(t+m_{Q}^{2}\right)\left(2 t+m_{Q}^{2}\right)}{t u_{1}}-2 m_{Q}^{2} \frac{t-5 m_{Q}^{2}}{t_{1}^{2}}+16 m_{Q}^{2}\left(\frac{u s-m_{Q}^{4}}{s t_{1} u_{1}}+\frac{m_{Q}^{2} t}{t_{1}^{3}}-\frac{m_{Q}^{4}}{s t_{1}^{2}}\right)+3\right) \\
& -C_{1}(s)\left(2 \frac{s s_{0} s_{1}}{t_{1} u_{1}}+\frac{s^{3}\left(4 Y+s^{2}\right)}{t_{1} u_{1} Y}-8 \frac{s^{2}\left(s-2 m_{Q}^{2}\right)}{Y}-2 s_{0}\right) \\
& -C(s) s\left(2 s \frac{4 m_{Q}^{2}-3 s}{Y}+\frac{s^{4}}{t_{1} u_{1} Y}+4 s_{1} \frac{s-2 m_{Q}^{2}}{t_{1} u_{1}}+2\right) \\
& -2 B(s) s \frac{s^{2}-2 s m_{Q}^{2}-2 t_{1} u_{1}}{s_{0} u_{1} t_{1}}-4 \ln \left(\frac{\lambda^{2}}{m_{Q}^{2}}\right)\left(2 \frac{m_{Q}^{2} s Y}{t_{1}^{2} u_{1}^{2}}-1\right) \\
& \left.-m_{Q}^{2} s \frac{11 t_{1} u_{1}-16 s m_{Q}^{2}}{t_{1}^{2} u_{1}^{2}}+m_{Q}^{4} \frac{t u_{1}+t_{1} u}{t t_{1} u u_{1}}+6+(t \leftrightarrow u)\right\},
\end{aligned}
$$

$$
\begin{aligned}
\mathcal{M}_{03}= & \frac{\alpha_{S} \alpha^{2} e_{Q}^{4}}{2 \pi s} \beta\left\{2 D(s, t)\left(\frac{s t s_{0}^{2}}{Y}-\frac{s\left(s-2 m_{Q}^{2}\right)\left(s t_{1}-8 m_{Q}^{2} t\right)}{t_{1} u_{1}}-2 m_{Q}^{2} s_{0}\right)\right. \\
& -C_{1}(t)\left(4 s_{0} \frac{s t-2 t_{1} m_{Q}^{2}}{Y}-4 s_{1} \frac{s-7 m_{Q}^{2}}{u_{1}}-4 m_{Q}^{2} \frac{s t-t_{1} m_{Q}^{2}}{t_{1}^{2}}\right) \\
& -2 B(t)\left(18 \frac{m_{Q}^{4}}{t_{1} u_{1}}-16 \frac{m_{Q}^{6}}{s t_{1} u_{1}}-m_{Q}^{2} \frac{\left(s-8 m_{Q}^{2}\right)\left(s t_{1}+2 s t-2 t_{1} m_{Q}^{2}\right)}{s t_{1}^{3}}\right. \\
& \left.-m_{Q}^{2} \frac{t_{1} u_{1}-11 t u_{1}-2 t t_{1}-t_{1} m_{Q}^{2}}{t t_{1} u_{1}}\right)-C(s)\left(\frac{s s_{0}^{2}}{Y}-s \frac{s s_{0}-8 m_{Q}^{2} s 1}{t_{1} u_{1}}-8 m_{Q}^{2}\right) \\
& +C_{1}(s) s s_{0}\left(\frac{s-2 m_{Q}^{2}}{t_{1} u_{1}}-\frac{s_{0}}{Y}\right)+8 \ln \left(\frac{\lambda^{2}}{m_{Q}^{2}}\right) \frac{m_{Q}^{2} s Y}{t_{1}^{2} u_{1}^{2}} \\
& -2 m_{Q}^{4} \frac{8 s^{2}+t_{1} u_{1}}{t_{1}^{2} u_{1}^{2}}+m_{Q}^{2}(t+u) \frac{m_{Q}^{4}+t_{1} u_{1}}{t t_{1} u u_{1}}+2 m_{Q}^{2} s \frac{t_{1}^{2}+u_{1}^{2}}{t_{1}^{2} u_{1}^{2}}-s \frac{s-15 m_{Q}^{2}}{t_{1} u_{1}}
\end{aligned}
$$




$$
+4+(t \leftrightarrow u)\} .
$$

Here $t_{2}=t+m_{Q}^{2}, s_{0}=s-4 m_{Q}^{2}$, and $s_{1}=s-m_{Q}^{2}$. The definitions and expressions for the scalar integrals are given in the Appendix A of [5]. It is worth mentioning that all functions $\mathcal{M}_{i}$ are expressed through four- and three-point functions and ultraviolet finite combinations of two-point functions.

\section{Real Gluon Emission}

The contribution of the real gluon emission to the total cross section is separated in two parts, soft gluon emission which cancels out the infrared divergences of virtual corrections and hard gluon emission. The cross section of the soft gluon emission can be reproduced in a factorized form as a product of Born level cross section and the infrared divergent factor:

$$
\frac{d \sigma^{\text {soft }}}{d t}=\frac{d \sigma^{\text {tree }}}{d t} R
$$

where

$$
\begin{aligned}
R= & \frac{8 \alpha_{s}}{3 \pi}\left\{\left[-1+\frac{1}{\beta}\left(1-\frac{2 m_{Q}^{2}}{s}\right) \ln \frac{1+\beta}{1-\beta}\right] \ln \frac{2 k_{c}}{\lambda}\right. \\
& +\frac{1}{2 \beta}\left(1-\frac{2 m_{Q}^{2}}{s}\right)\left[\operatorname{Sp}\left(\frac{-2 \beta}{1-\beta}\right)-\operatorname{Sp}\left(\frac{2 \beta}{1+\beta}\right)\right] \\
& \left.+\frac{1}{2 \beta} \ln \frac{1+\beta}{1-\beta}\right\},
\end{aligned}
$$

Here $k_{c}$ is the soft photon energy cut and the velocity $\beta$ is defined in Section 2 . The dependence on the gluon mass $\lambda$ is exactly canceled while adding soft gluon emission part and virtual corrections for any sets of Stokes parameters. As can be seen from Eqs. (6) and (8) the term proportional to the product $\xi_{1}^{(1)} \xi_{2}^{(2)}$ appears only in the virtual part and therefore should be infrared finite, although one-loop scalar functions $D(s, t), D(s, u)$ and $C_{1}(s)$ do contain infrared logarithms. One can easily check that infrared divergent contributions from these scalar functions cancel each other in the $\mathcal{M}_{12}$-term of Eq. 8 .

The hard gluon emission part is also calculated using FORM program. The expressions for the squared matrix elements are lengthy and we do not reproduce them in the paper. The integration over three-particle phase space is done by using Monte-Carlo integration routine VEGAS [11]. After adding the soft and hard gluon emission cross sections the final numerical results do not depend on the imposed energy cut of emitted gluon, $k_{c}$. Special care is taken to handle sharp peaks of the cross section while the gluon is soft or is emitted along the quark or antiquark three momenta (see detailed discussion in [12]). These peaks correspond to the infrared and collinear singularities in the case of massless fermions and in our case become essential when mass of quark is small compared to the c.m.s. energies of photons. To arrange the infrared and collinear singularities along the integration axis we take the gluon energy, denominator of the quark propagator, polar angles of quark and antiquark and azimuthal angle of reaction as integration variables. Integration over azimuthal angle is necessary in the case of linearly polarized photons, as in this case the cross section does depend on the $\phi$ angle. The infrared singularity lies on the axis of integration over gluon energy, whereas collinear singularities are located along the axis 
of quark propagator. There are also additional peaks when quark or antiquark are produced at zero angle with respect to the beam direction. Such singularities are treated by integration over the polar angles of quark and antiquark.

\section{Results and Discussion}

As was mentioned above, the helicity cross sections $\sigma\left(J_{z}=0\right), \sigma\left(J_{z}=2\right)$ for the heavy quark pair production in the circularly polarized photon-photon collisions were considered in [5, 6]. In this paper we mainly present numerical results for the production of heavy quark-antiquark pair for the linearly polarized photons. We consider two cases of linear polarizations of initial photons, when $\Delta \gamma=0$ and $\pi / 2$, where $\Delta \gamma$ is the angle between the directions of polarization vectors of the photons. The $\Delta \gamma=0, \pi / 2)$ correspond to the collision of linearly polarized photons with parallel and perpendicular polarizations, respectively. For the measurements of the Higgs boson parity it is necessary to consider collisions of linearly polarized photons in order to measure the polarization asymmetry [3]

$$
A=\frac{\sigma_{\perp}-\sigma_{\|}}{\sigma_{\perp}+\sigma_{\|}}
$$

In fact if inclusive two-jet final states are studied then after averaging over azimuthal angles and spins of the final particles only three independent cross sections remain for arbitrary polarization states of initial photons. These independent cross sections can be taken as $\sigma_{t o t}, \sigma\left(J_{z}=0\right)-\sigma\left(J_{z}=2\right)$ and $\sigma_{\perp}-\sigma_{\|}$[1].

For the study of the Higgs boson signal in photonphoton collisions [4, 5, 6] it was essential that the background from $b \bar{b}$ quark production is suppressed by a factor of $m_{b}^{2} / s$ for $J_{z}=0$ at the Born level. However at the next-to-leading order the cross section of the $b \bar{b} g$ production for $J_{z}=0$ is not suppressed any more. Therefore experimental cuts selecting only two-jet final states were important to suppress the $b \bar{b} g$ background [甘, 5, 6]. In this Section we show, that the difference of $\sigma_{\perp}-\sigma_{\|}$is suppressed by a factor of $m_{Q}^{2} / s$ even at the next-to-leading order.

The cross section for heavy quark pair production

can be cast in the form [13]:

$$
\sigma_{\gamma \gamma \rightarrow Q \bar{Q}(g)}=\frac{\alpha^{2} Q^{4} N_{c}}{s}\left(f_{\gamma \gamma}^{(0)}+\frac{4}{3} \frac{\alpha_{s}}{\pi} f_{\gamma \gamma}^{(1)}\right)
$$

where the functions $f_{\|, \perp}^{(0,1)}$ depend on the dimensionless variable $s /\left(4 m_{Q}^{2}\right)$ only. The numerical values of the functions $f_{\|, \perp}^{(0,1)}$ are presented in the Figure 1 and Table I. Because of the Sommerfeld rescattering correction, the function $f_{\perp}^{(1)}$ is nonzero at the threshold, as one can see from the table I. In the both cases, $\Delta \gamma=0$ and $\Delta \gamma=\pi / 2$, the functions corresponding to QCD corrections are positive and rising at high energies. Taking the average of values of these two functions one obtains the corresponding function for the unpolarized cross section. Our results are in agreement with previous calculations of QCD corrections for heavy quark-antiquark production 
Table 1: $f^{(0,1)}$ for different polarization states of the photons as a function of dimensionless parameter $s /\left(4 m_{Q}^{2}\right)$.

\begin{tabular}{|c|c|c|c|c|c|c|}
\hline$\frac{s}{4 m_{Q}^{2}}$ & $f_{\perp}^{(0)}$ & $f_{\|}^{(0)}$ & $f_{\text {unpol }}^{(0)}$ & $f_{\perp}^{(1)}$ & $f_{\|}^{(1)}$ & $f_{\text {unpol }}^{(1)}$ \\
\hline 1 & 0.0 & 0.0 & 0.0 & 124.0 & 0.0 & 62.0 \\
4 & 28.6 & 24.9 & 26.7 & 34.3 & 54.8 & 44.5 \\
9 & 36.6 & 35.0 & 35.8 & 54.6 & 70.4 & 62.5 \\
16 & 42.5 & 41.6 & 42.1 & 93.4 & 104 & 98.6 \\
25 & 47.3 & 46.8 & 47.1 & 136 & 144 & 140 \\
100 & 63.4 & 63.3 & 63.3 & 339 & 340 & 339 \\
400 & 80.4 & 80.3 & 80.3 & 662 & 674 & 668 \\
2500 & 103.2 & 103.2 & 103.2 & 1352 & 1359 & 1355 \\
\hline
\end{tabular}

in unpolarized photon-photon collisions [5, 13, 14]. As in the case of Born level functions $f_{\|}^{(0)}$ and $f_{\perp}^{(0)}$, in the asymptotic regime the difference between $f_{\|}^{(1)}$ and $f_{\perp}^{(1)}$ vanishes and each of the function tends to the unpolarized one, $f_{\text {unpol }}^{1}$. Such an asymptotic behavior of the corrections can be understood considering the helicity amplitudes for massless quarks. The difference of the cross sections with parallel and orthogonal polarized photons can be expressed via the interference term of the following helicity amplitudes

$$
\begin{aligned}
\Delta \sigma(\gamma \gamma \rightarrow Q \bar{Q})=\sigma_{\perp}-\sigma_{\|} \simeq & \operatorname{Re} \sum M_{++}^{\text {Born }}(\gamma \gamma \rightarrow Q \bar{Q}) M_{--}^{\text {one-loop* }}(\gamma \gamma \rightarrow Q \bar{Q}) \\
& +\operatorname{Re} \sum M_{++}^{\text {one-loop }}(\gamma \gamma \rightarrow Q \bar{Q}) M_{--}^{\text {Born* }}(\gamma \gamma \rightarrow Q \bar{Q}) \\
& +\operatorname{Re} \sum M_{++}^{\text {Born }}(\gamma \gamma \rightarrow Q \bar{Q} g) M_{--}^{\text {Born* }}(\gamma \gamma \rightarrow Q \bar{Q} g)
\end{aligned}
$$

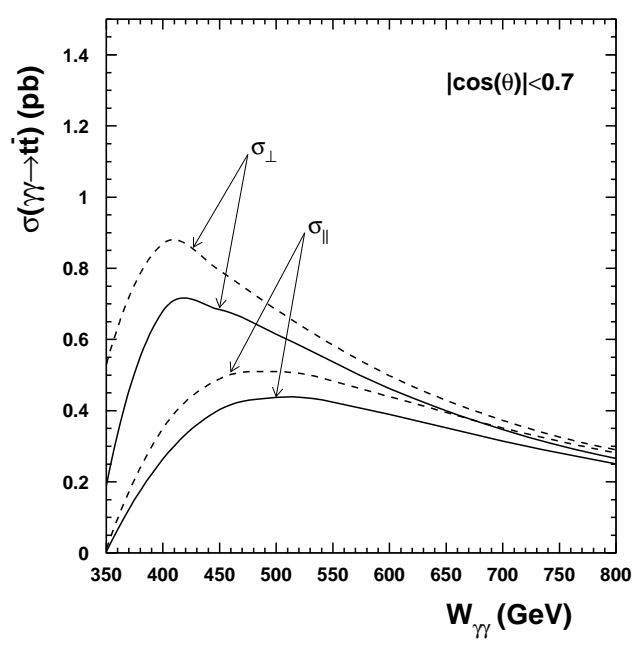

Figure 2: The $t \bar{t}$ production cross section for parallel and orthogonal polarized photon collisions versus c.m.s. energy of photons; the solid lines correspond to the Born cross section and the dashed lines to the cross sections with QCD corrections. here the sum over the helicities of the final state particles $Q \bar{Q}(g)$ is implied. The Born amplitude of the $Q \bar{Q}$ pair production in the photon-photon collisions is known to vanish like $m_{Q}^{2} / s$ for equal photon helicities and massless quarks [15, 5, 6]. Therefore first two terms in the Eq. 19 vanish in the high energy limit. In addition, helicity amplitudes for the process of massless $Q \bar{Q}$ pair production with the additional gluon emission $\gamma \gamma \rightarrow Q \bar{Q} g$ identically vanish for photon and gluon helicities $\lambda_{1}=\lambda_{2}=-\lambda_{g}= \pm 1$ and arbitrary quark helicities [15]. Consequently, in the third term of the Eq. 19 amplitudes $M_{++}^{\text {Born }}(\gamma \gamma \rightarrow Q \bar{Q} g)$ and $M_{--}^{\text {Born }}(\gamma \gamma \rightarrow Q \bar{Q} g)$ are nonzero only in the case when emitted gluons have different polarizations. Therefore there is no interferention between corresponding amplitudes and third term of Eq. 19 term also vanishes at high energies. As result, the difference of the cross sections for $(Q \bar{Q})$-pair production, $\Delta \sigma(\gamma \gamma \rightarrow Q \bar{Q})$ is suppressed by a factor of $O\left(m_{Q}^{2} / s\right)$. For $b \bar{b}$ production the relative difference of the cross section for parallel and orthogonal polarized photons is less than $1 \%$ for 
$\sqrt{s} \geq 200 \mathrm{GeV}$, i.e. in the whole range of the PLC energies. On the other hand, for top-antitop production there is no strong suppression of $\Delta \sigma$ by the mass of quark at energies $\sqrt{s} \simeq 500 \div 800$ $\mathrm{GeV}$. The production cross sections for $t \bar{t}$-pair production are illustrated in the Figure 2 for different helicity states of initial photons. The usual cut for suppression the Higgs background is imposed, $|\cos \theta|<0.7$. The solid lines correspond to the Born level cross sections and the dashed lines to the QCD corrected ones. As one can see from Figure 2 the corrections are large near the threshold and decrease very rapidly with increasing the photon-photon c.m.s energy, $W_{\gamma \gamma}$.

In the Figure 3 a the difference of two cross sections, $\Delta \sigma(\gamma \gamma \rightarrow t \bar{t})$, is shown. The correction
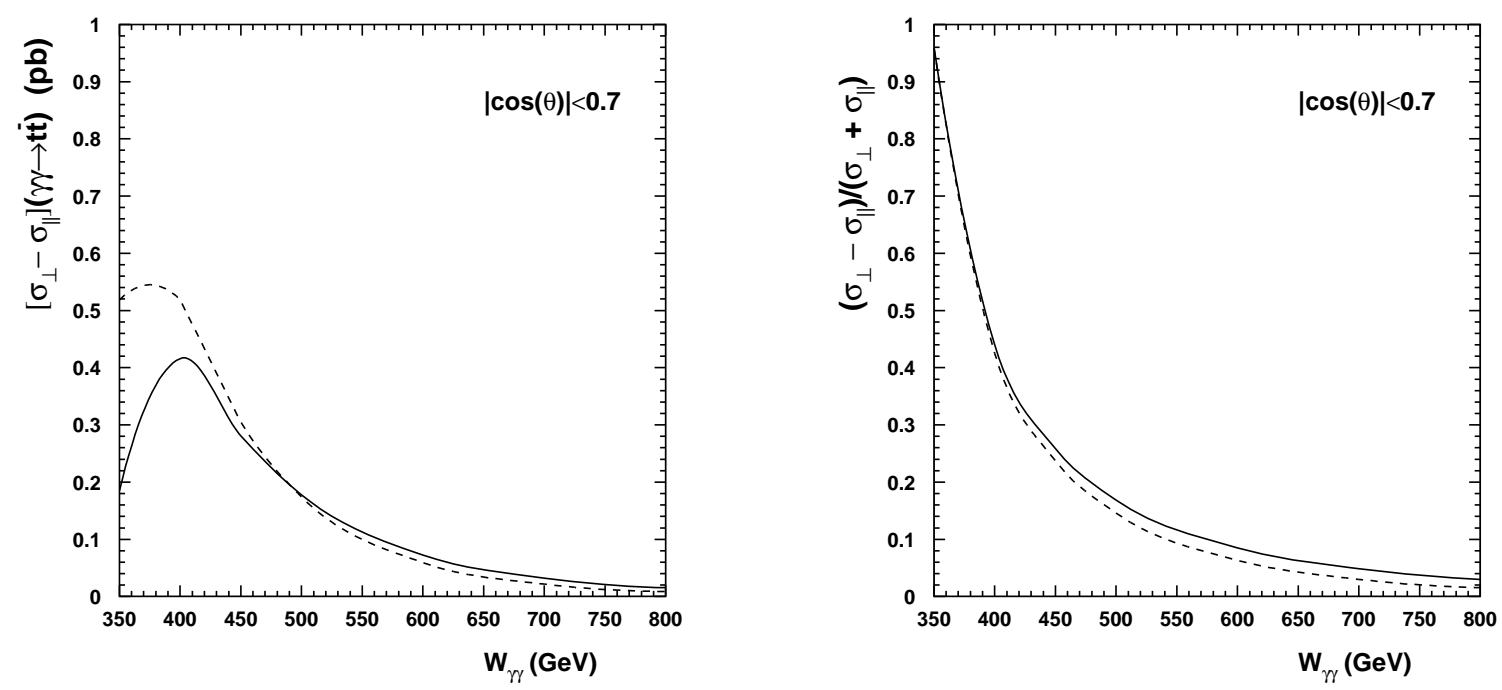

Figure 3: The difference of top-antitop production cross section, $\Delta \sigma(\gamma \gamma \rightarrow t \bar{t})$; a) the absolute value of $\Delta \sigma, \mathrm{b})$ the relative value of $\Delta \sigma$. The definition of lines is the same as in the previous figure.

to the $\Delta \sigma$ are rather large near the threshold, up to $W_{\gamma \gamma} \simeq 400 \mathrm{GeV}$, and decreases rapidly. However, the asymmetry, $\left(\sigma_{\perp}-\sigma_{\|}\right) /\left(\sigma_{\perp}+\sigma_{\|}\right)$gets only small corrections in the whole range of energies.

The azimuthal asymmetry of heavy quark pair production is the specific effect which occurs only at one-loop level. The relevant term in Eq. 8 proportional to imaginary unit $i$ corresponds to the scattering when one photon is polarized linearly and the other circularly, $\xi_{1}^{(1)}= \pm 1$ and $\xi^{(2)}= \pm 1$ or other way round (all other Stokes parameters are zero). This term gives the contributions with opposite signs to the cross section being integrated in two different ranges of azimuthal angle, $-\pi / 2<\phi<0$ and $0<\phi<\phi / 2$. The value $\phi=0$ corresponds to the direction of polarization vector of linearly polarized photon. It is obvious that the total contribution of $\mathcal{M}_{12}$-term to the cross section is zero. We define the azimuthal asymmetry of $(Q \bar{Q})$-pair production in the linearly polarized photon scattering off circularly polarized one in the following way

$$
A_{\phi}=\frac{\sigma\left(-\frac{\pi}{2}<\phi<0\right)-\sigma\left(0<\phi<\frac{\pi}{2}\right)}{\sigma\left(-\frac{\pi}{2}<\phi<0\right)+\sigma\left(0<\phi<\frac{\pi}{2}\right)} .
$$

This asymmetry can be sizeable only for top-antitop production at PLC energies, while for the $b \bar{b}$ pair production azimuthal asymmetries are also suppressed by factor $O\left(m_{b}^{2} / s\right)$. The expectations for the $t \bar{t}$-pair production asymmetry are shown in Figure 3 . The effect is $1.5 \%$ at energies of the PLC. 


\section{Conclusion}

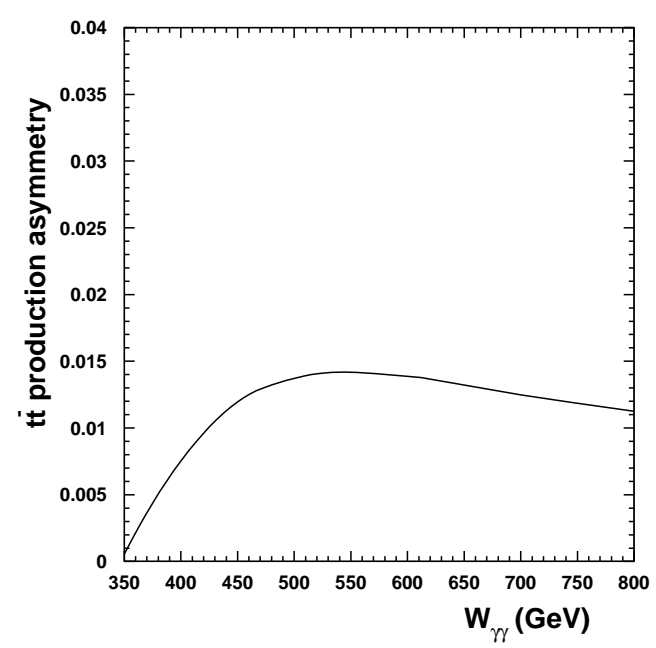

Figure 4: The asymmetry of top-antitop production in the photon-photon collisions.

In the presented paper we derived the compact expressions for the $\alpha_{s}$-corrections to the squared matrix element $\gamma \gamma \rightarrow Q \bar{Q}$ for the general case of initial photon polarizations. The total cross sections up to the order $\alpha^{2} \alpha_{s}$ are calculated, which are given by the sum of the tree level cross section, contribution of the interference term between the QCD one-loop $\alpha_{s}$-correction and tree level amplitude and tree level cross sections of quark pair production accompanied by the real gluon emission. The numerical results are obtained for heavy quark pair production cross sections in the case of linearly polarized photon collisions. The difference of the cross sections of heavy quark pair production for parallel and perpendicular polarized photon collisions is suppressed by factor $m_{Q}^{2} / s$. We show that the QCD correction for the $b \bar{b}$ production asymmetry is less than $1 \%$ in the whole energy range of the PLC and practically does not change the background for the measurement of $C P$ parity of Higgs boson. At the same time, there is no such suppression for top-antitop production due to the large mass of top quark. The relevant cross sections for $t \bar{t}$-pair productions are calculated. The QCD corrections are large near the threshold and decrease rapidly with increasing the c.m.s energy of colliding photons.

We also calculated the azimuthal asymmetries for $t \bar{t}$ production. This effect arises only at one-loop level and the asymmetry is about $1.5 \%$ in the energy range of the PLC.

\section{Acknowledgments}

We would like to thank J.I. Illana for useful discussions.

\section{References}

[1] I.F. Ginzburg, G.L. Kotkin, V.G. Serbo and V.I. Telnov, Pis'ma ZhETF 34, 514 (1981); Nucl. Instr. Methods 205, 47 (1983);

I.F. Ginzburg, G.L. Kotkin, S.L. Panfil, V.G. Serbo and V.I. Telnov, Nucl. Instr. Methods 219, 5 (1984).

[2] J.F. Gunion, H.E. Haber, G .Kane and S. Dawson, The Higgs hunter's guide, AddisonWesley Reading, MA, 1990.

[3] B. Grzadkowski and J.F. Gunion, Phys. Lett. B294, 261 (1992);

J.F. Gunion and J. Kelly, Phys. Lett. B333, 110 (1994);

V. Barger, K. Cheung, A. Djouadi, B.A. Kniehl, P.M. Zerwas, Phys. Rev. D49, 79 (1994); 
M. Krämer, J. Kün, M.I. Stong and P.M. Zerwas, Z. Phys. C64, 21 (1994);

G.J. Gounaris and G.P. Tsirigoti, Phys. Rev. D56, 3030 (1997).

[4] D.L. Borden, D. A. Bauer, D. O. Caldwell, Phys. Rev. D48, 4018 (1993)

D.L. Borden, V.A. Khoze, W.J. Stirling, J. Ohnemus, Phys. Rev. D50, 4499 (1994).

[5] G. Jikia and A. Tkabladze, Proc. of the Workshop on gamma-gamma colliders, March 2831, 1994, Lawrence Berkeley Laboratory, Nucl. Instr. and Meth. A355, 81-83, (1995); Phys. Rev. D54, 2030 (1996).

[6] B. Kamal, Z. Merebashvili and A.P. Contogouris, Phys. Rev. D51, 4808 (1995).

[7] V.S. Fadin, V.A. Khoze and A.D. Martin, Phys. Rev. D56, 484 (1997).

[8] M. Melles, W.J. Stirling, Phys. Rev. D59, 94009 (1999); Eur. Phys. J. C 9, 101 (1999);

M. Melles, W.J. Stirling, V.A. Khoze, Phys. Rev. D61, 54015 (2000).

[9] A. Denner, S. Dittmaier, Nucl. Phys. B540, 58 (1999).

[10] J.A.M. Vermaseren, Symbolic Manipulation with FORM, published by CAN, Kruislaan 413, 1098 SJ Amsterdam, 1991, ISBN 90-74116-01-9.

[11] G.P.Lepage, J. Comput. Phys. 27, 192 (1978).

[12] J. Fujimoto, M. Igarashi, N. Nakazawa, Y. Shimizu and K. Tobimatsu, Prog. Theor. Phys. Suppl. 100, 1 (1990).

[13] J.H. Kühn, E. Mirkes, J. Steegborn, Z. Phys. C57, 615 (1993).

[14] M. Drees, M. Krämer, J. Zunft, and P.M. Zerwas, Phys. Lett. B306, 371 (1993).

[15] R. Gastmans and Tai Tsun Wu, The Ubiquitous Photon: Helicity method for QED and QCD, Oxford: Clarendon, 1990. 